\title{
THE MORPHOMETRIC PARAMETERS OF THE PARENCHYMAL AND STROMAL LIVER COMPONENTS OF HEALTHY NEWBORNS FROM HEALTHY MOTHERS WITH A PHYSIOLOGICAL COURSE OF PREGNANCY
}

D0I: 10.36740/WLek202009201

\author{
Sergiy 0. Sherstiuk, Stanislav I. Panov, Tetiana I. Liadova, Oleksij I. Tsivenko, Liudmila L. Sherstiuk \\ V.N. KARAZIN KHARKIV, NATIONAL UNIVERSITY, KHARKIV, UKRAINE
}

\begin{abstract}
The aim: To determine the morphometric parameters of the parenchymal and stromal liver components of healthy newborns.

Material and methods: The morphometric investigation included 45 liver tissue biopsies of healthy newborns. All morphometric parameters of the parenchymal and stromal liver components were calculated using the Avtandilov microscopic morphometric grid. It was inserted into the microscope ocular tube with a total $\times 200$ microscope magnification. The number of points that were found on the corresponding types of parenchymal and stromal liver components was calculated. In every case, it was selected 10 random microscopic areas and then all data were obtained, calculated and presented as percentages.

Results: Morphometric parameters of hepatocytes: mononuclear hepatocytes $-93.5 \pm 7.1 \%$, two-nuclear hepatocytes $-6.5 \pm 1.2 \%$, BMHC (bi-/mononuclear hepatocytes coefficient) $-0.06 \pm 0.01$, hepatocytes with fat vacuoles $-0.5 \pm 0.2 \%$. Parenchymal and stromal liver components: parenchyma $-74.2 \pm 4.3 \%$, stroma (including blood vessels and bile ducts) $-25.8 \pm 2.6 \%, \mathrm{SPI}$ (stroma/parenchyma index) $-0.34 \pm 0.01$. Morphometric parameters of all of the liver components: hepatocytes $-74.2 \pm 4.3 \%$, portal tracts $-3.1 \pm 0.6 \%$, central veins $-9.3 \pm 1.4 \%$, sinusoids $-10.5 \pm 1.3 \%$, bile ducts $-2.9 \pm 0.2 \%$. Expression level parameters: fibronectin $-17.3 \pm 2.5 \%$, collagen type I $-9.7 \pm 1.9 \%$, collagen type III $-10.1 \pm 0.9 \%$, collagen type IV $-5.9 \pm 0.2 \%$. Parameters of liver fibrosis biomarkers: APRI (index) $-0.19 \pm 0.01$, a FIB-4 (index) $-0.022 \pm 0.001$.

Conclusions: The morphometric parameters of the parenchymal and stromal liver components of healthy newborns can be used as a control group in the study of any pathological conditions of the liver of newborns.
\end{abstract}

KEY WORDS: liver, newborn, liver parenchyma, liver stroma

Wiad Lek. 2020;73(9 p. II):1895-1899

\section{INTRODUCTION}

Unfortunately, timely diagnosis of liver disease in newborn infants remains an urgent problem today, since various disorders of the liver often have virtually the same initial clinical features, or they generally have a latent course. Since liver dysfunction is progressive, early detection of pathology allows time to initiate appropriate therapeutic measures and significantly improve the prognosis [1]. The role of the pathomorphologist in addressing common problems of liver disease in children is likely to change in the future with the progresses of molecular genetic revolution. However, even when biochemical, microbiological, and immunological laboratories already provide essential diagnostic information, an informed morphological interpretation of liver disease in children continues to be an important method of diagnosis [2]. Primary interpretation of liver disease in children may occur through clinical and laboratory data, but differential diagnosis and prognosis of liver disease require more accurate morphological data, and this primarily concerns the assessment of the stromal-parenchymal component of the liver [3]. In order to correctly and reliably evaluate the quality of the stromal-parenchymatous component of the liver of a newborn baby and to correctly interpret the presence or absence of liver pathology, information on the normal rates of stroma and liver parenchyma in healthy newborn infants should be compared. The study of world and national resourses showed the absence of such data, so there is a need to provide such information to the scientific community and practitioners to optimize diagnosis of liver disease in children.

\section{THE AIM}

Study of morphometric parameters of stromal-parenchymatous component of liver of healthy full-term newborns from healthy mothers with physiological course of pregnancy, with further provision of the obtained data to the scientific community and practitioners to optimize the process of diagnosis of liver pathology in children.

\section{MATERIALS AND METHODS}

Investigated 45 biopsies of the liver tissue of healthy term infants of 37-40 weeks gestational age born to healthy 
mothers with physiological pregnancy and died due to severe head injury, or premature detachment of normally situated placenta during delivery; life expectancy in all cases did not exceed 24 hours. The autopsiy material research was conducted according to the requirements of «Instruction on conducting autopsies» (Ministry of Health of Ukraine order № 6 from 17.01.1995); the requirements, norms and standard provisions on ethics of the Ministry of Health of Ukraine № 690 from 23.09.2009 p.; «The procedure for the removal of biological objects from the dead, whose bodies are subject to forensic and pathoanatomical research, for scientific purposes» (2018). The material was collected during the period from 2010 to 2018 in pathoanatomical department of Kharkiv Regional Perinatal Center.

For morphological study liver slices were fixed in $10 \%$ solution of neutral formalin and then subjected to standard processing through liquid Nikiforov (96\% alcohol and diethyl ether in the ratio 1: 1), chloroform and then paraffinization.

The recived blocks were sliced intro pieces of $4-5 \mu \mathrm{m}$ thick by Microm HM - 340 microtome. The obtained samples were coloured with hematoxylin and eosin as well as using method Mallory.

Regenerative activity of liver parenchyma was evaluated by the number of binucleated and mononucleated hepatocytes. The number of binucleated / mononucleated hepatocytes was calculated using the Avtandilov microscopic morphometric grid, which consisted of 100 equidistant points and was inserted into the microscope eyepiece with $\times 200$ magnification of the microscope. The number of dots that occurred in single and dual hepatocytes was counted. Each sample was studied in 10 randomly selected fields of view, and the data were calculated and presented as a percentage [4]. Also, the calculated rate of binucleated / mononucleated hepatocytes (RBMH) by the formula:

\section{RBMH = Number Of Binuclear Hepatocytes (\%) / \\ Number Of Mononuclear Hepatocytes (\%)}

For morphometric assessment of the stromal, parenchymatous, vascular and biliary components of the liver, was also used Avtandilov microscopic morphometric grid, which consisted of 100 equidistant points and was inserted into the microscope eyepiece with $\times 200$ magnification of the microscope. The number of points that fell on the stromal (portal tracts, septa), parenchymatous (hepatocytes), vascular (portal vessels, central veins, sinusoids) and biliary (bile ducts) components of the liver were calculated. Each sample was examined in 10 randomly selected fields of view, and then the data obtained were calculated and presented as a percentage. Also, stromal-parenchymal index (SPI) was calculated by the formula:

\section{SPI = Stromal Index / Parenchymal Index}

Immunohistochemical features of the components of the liver were studied using indirect immunoperoxidase method on paraffinized sample of 3-5 microns thick. In all cases, each sample was studied in 10 randomly selected fields of view, and positive signals (positive expression in the form of a brown color) were measured by Avtandylov microscopic morphometric grid, which consisted of 100 equally equidistant points was inserted into the eyepiece eyepiece with $\times 200$ magnification of the microscope. The number of dots that occurred in the positive signal was counted, and the data were calculated and presented as a percentage. The samples were studied using a BX43 optical microscope (Olympus Corporation, Tokyo, Japan) and the expression was analyzed using a quantitative image analysis system (Image-Pro Plus 6.0; Media Cybernetics Inc., Rockville, MD, USA).

Fibronectin expression level fibronectins antibodies (mouse polyclonal; 1: 100; sc-8422; Santa Cruz Biotechnology, Inc.) were usied. The blocks were heated for 60 minutes at $60^{\circ} \mathrm{C}$, deparaffinized with xylene and washed in alcohols with a decrease in their concentration. Antigens were obtained with citrate buffer $(\mathrm{pH}$ 6.0) in a microwave oven for 5 minutes. After being processed in $1.5 \% \mathrm{H}_{2} \mathrm{O} 2$ at $37^{\circ} \mathrm{C}$ for 30 minutes to block endogenous peroxidase activity, the samples were incubated with primary antibodies overnight at $4^{\circ}$ $\mathrm{C}$ and then incubated with secondary antibodies (PV-9002; OriGene Technologies, Inc., Beijing, China) for 30 minutes at room temperature. Finally, samples were couloured using diaminobenzidine followed by hematoxylin contrast.

To assess the level of expression of collagen types I and III collagen using antibodies against type I and III (polyclonal rabbits, 1: 600; Abcam, Cambridge, UK). The blocks were heated for 60 minutes at $60^{\circ} \mathrm{C}$, deparaffinized with xylene and washed in alcohols with decreasing concentration. The preparations were first pre-incubated in $3 \% \mathrm{H} 2 \mathrm{O} 2$ solution for 10 minutes at room temperature to block endogenous peroxidase activity, and then the antigen was extracted with citrate buffer ( $\mathrm{pH} 6.0$ ) in a microwave oven at $95-98^{\circ} \mathrm{C}$ for 12 minutes, followed by incubation for 5 minutes at room temperature to block non-specific background staining. Then, incubated at room temperature for 30 minutes with primary rabbit polyclonal antibodies against type I and III collagen. The obtained preparations were washed four times with buffer, treated with streptavidin, peroxidase and chromogen DAB. Finally, the sections were stained using diaminobenzidine followed by hematoxylin contrast.

To assess the level of expression of type IV collagen used antibodies against type IV collagen (polyclonal mice; $1: 100$; Leica Biosystems, Newcastle, UK: PHM-12, UK). The blocks were heated for 60 minutes at $60^{\circ} \mathrm{C}$, deparaffinized with xylene and washed in alcohols with decreasing concentration. Antigens were obtained with citrate buffer $(\mathrm{pH}$ 6.0) in a microwave oven for 5 minutes and then cooled at room temperature for 20 minutes. After processing 3\% solution of $\mathrm{H} 2 \mathrm{O} 2$ at $37^{\circ} \mathrm{C}$ for 30 minutes to block the endogenous activity of peroxidase, sections were incubated with primary antibodies overnight at $4^{\circ} \mathrm{C}$, and then incubated with secondary antibody (K-ASSAY Collagen Type IV Staining Kit, Kamiya Biomedical Company, USA) for 30 minutes at room temperature. Finally, sections were stained using diaminobenzidine followed by hematoxylin contrast.

To assess the presence of liver fibrosis, a non-invasive analysis of two biomarkers of liver fibrosis in blood APRI and FIB-4 was performed. Indicators of biomarkers of fibrosis were calculated according to officially published 


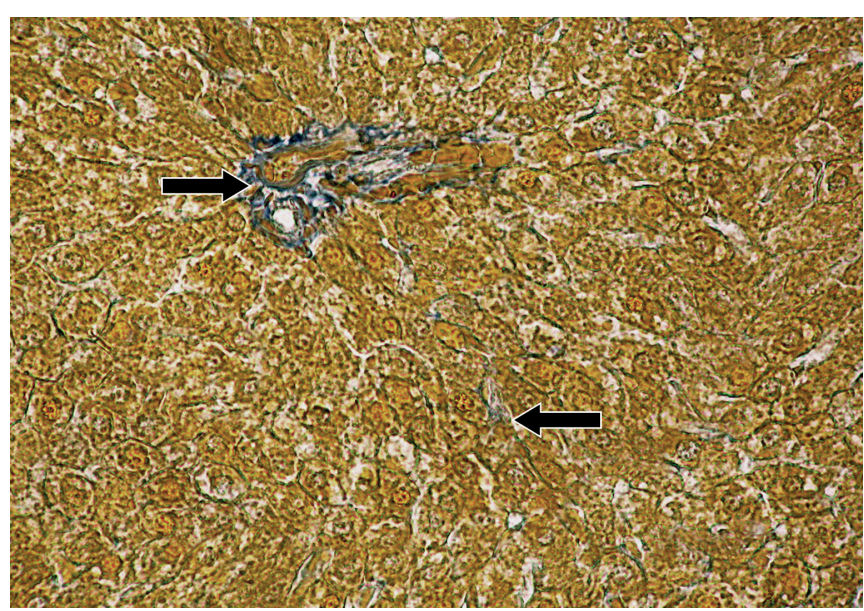

Fig. 1. The liver of a healthy newborn baby. Conventional histoarchitectonics of the liver, normal development of the stromal component (arrows). Mallory coloring. $\times 200$.

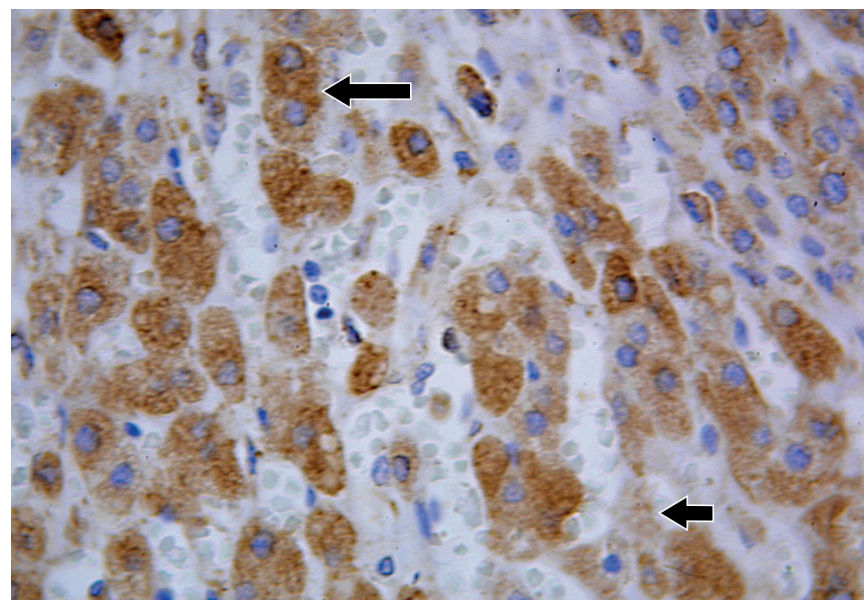

Fig. 2. The liver of a healthy newborn baby. Fibronectin matrix protein in the cytoplasm of hepatocytes (long arrow) and in Disse spaces in the course of sinusoids (short arrow). Indirect immunoperoxidase method. $\times 400$.

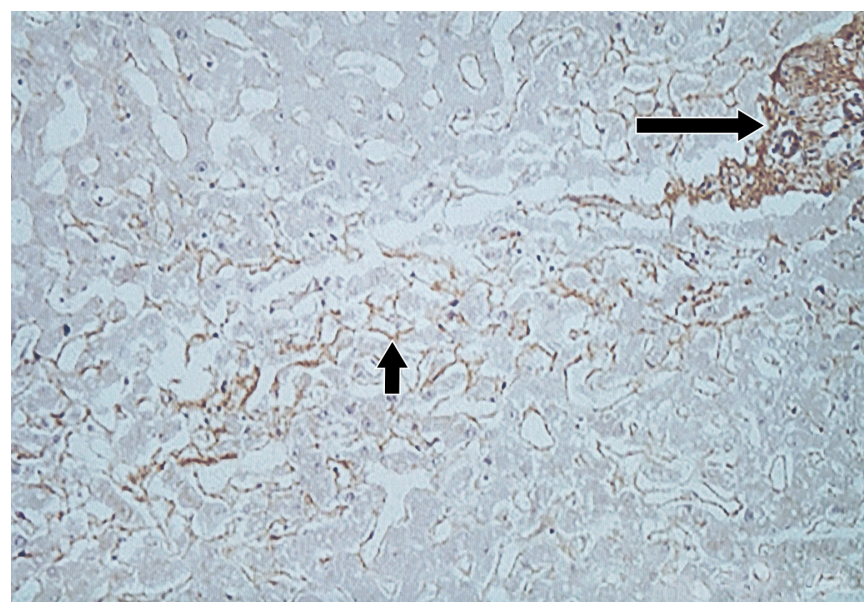

Fig. 3. The liver of a healthy newborn baby. Expression of type I collagen in the stroma of the portal tracts (long arrow) and in the Disse spaces along sinusoids (short arrow). Indirect immunoperoxidase method. $\times 200$.

recommendations $[5,6,7,8]$ using the following formulas: APRI $=[$ AST $(I U / L) /$ AST $($ ULN $)(I U / L) /$ Platelet Count $\left.\left(10^{9} / \mathrm{L}\right)\right] \times 100$,

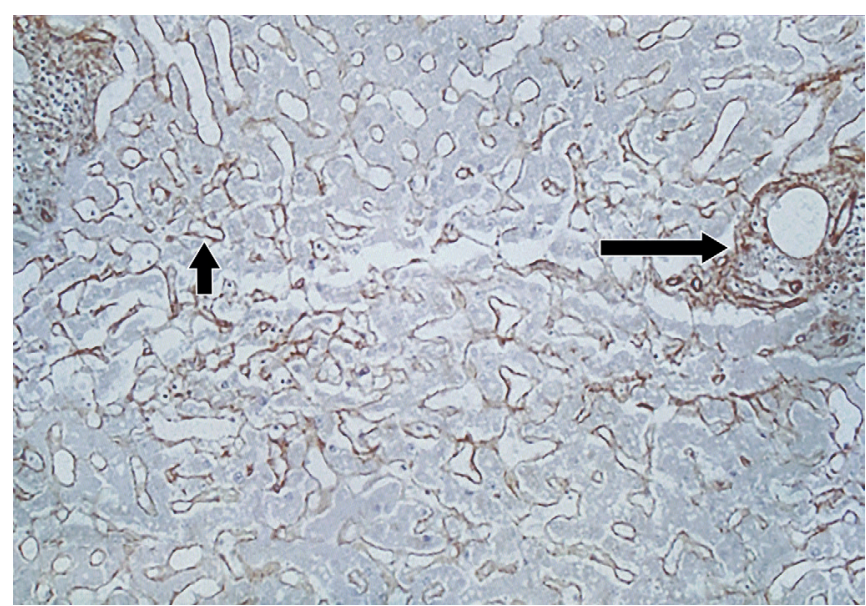

Fig. 4. The liver of a healthy newborn baby. Expression of type III collagen in the stroma of portal tracts (long arrow) and in Disse spaces along sinusoids (short arrow). Indirect immunoperoxidase method. $\times 200$.

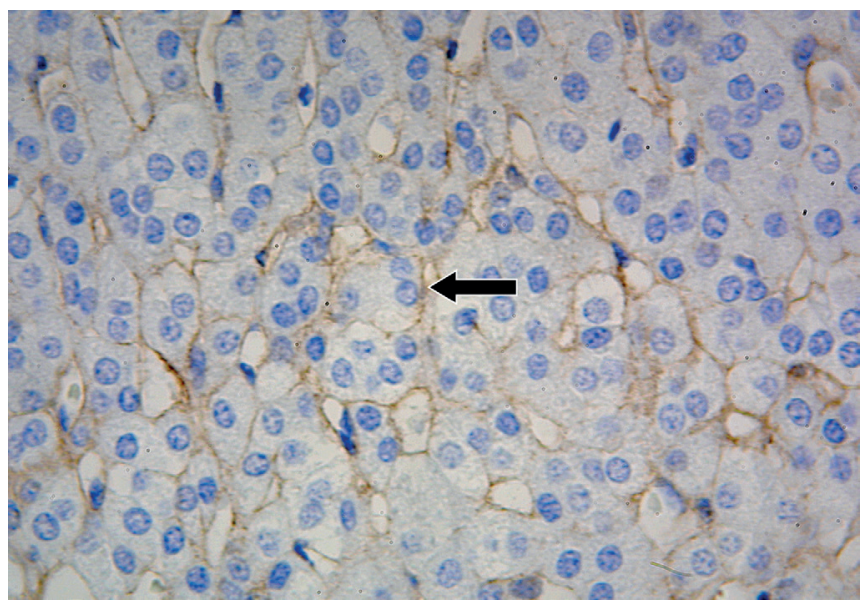

Fig. 5. The liver of a healthy newborn baby. Expression of type IV collagen in Disse spaces along sinusoids (arrow). Indirect immunoperoxidase method. $\times 400$.

AST - aspartate aminotransferase; AST ULN (Upper Limit of Normal) - aspartate aminotransferase (40 IU/L).

FIB-4 $=[$ Age $($ years $) \times$ AST $($ IU $/ L)] /[$ Platelet Count $\left(10^{9} / \mathrm{L}\right) \times \sqrt{ }$ ALT $($ IU/L) $]$

ALT - alanine aminotransferase; AST - aspartate aminotransferase.

Statistical processing of the results was performed using the standard statistical software package «STATISTICA 10.0» and «MS Excel». For derived indicators calculated arithmetic mean (M), the average standard error $(\mathrm{m})$. To assess the degree of reliability of differences between groups using U-criterion Mann-Whitney.

\section{RESULTS}

In the macroscopic study liver was elastic to the touch and had excellent thin translucent capsule. On the cross section of the liver tissue was red-brown, homogeneous, with properly developed and unevenly full-blooded hepatic veins. Microscopically was observed the normal radiarna-beam structure, normal sinusoids and central veins, and a few small sites of extramedullary hematopoiesis. 
Hepatocytes had slightly granular eosinophilic cytoplasm and round basophilic nucleus. The morphometric parameters of hepatocytes were as follows: mononuclear hepatocytes - $93.5 \pm 7.1 \%$, binuclear hepatocytes - $6.5 \pm 1.2 \%$, RBMH (binuclear / mononuclear hepatocytes coefficient) - $0.06 \pm$ 0.01 , hepatocytes with fat vacuoles $-0.5 \pm 0.2 \%$.

The portal stroma was developed normally and consisted with collagen fibers.

Stromal-parenchymal indices of liver were as follows: parenchyma - 74.2 $\pm 4.3 \%$, stroma (including vessels and bile ducts) - $25.8 \pm 2.6 \%$, SPI (stromal-parenchymal index) $-0.34 \pm 0.01$.

The morphometric parameters of all components of the liver were as follows: hepatocytes $-74.2 \pm 4.3 \%$, portal tracts - $3.1 \pm 0.6 \%$, central veins $-9.3 \pm 1.4 \%$, sinusoids - $10.5 \pm$ $1.3 \%$, bile ducts $-2.9 \pm 0.2 \%$. Across the liver parenchyma had a moderate amount of fibronectin matrix protein in the cytoplasm of hepatocytes and in the Disse spaces along of sinusoids.

Tape I and III had moderate expression in the stroma of the portal tracts. Also, those types of collagen were detected in Disse spaces along of sinusoids in a small amount, which was documented by their weak expression.

There was a moderate amount of type IV collagen in the Disse spaces along sinusoids, which is normal major component of basal membranes.

The levels of expression of fibronectin, type I, III and IV collagens in the stromal component of the liver were as follows: fibronectin - $17.3 \pm 2.5 \%$, type I collagen - 9.7 $\pm 1.9 \%$, type III collagen $-10.1 \pm 0.9 \%$, type IV collagen $-5.9 \pm 0.2 \%$.

The level of ALT in the blood of newborns averaged 12.6 $\pm 2.2 \mathrm{IU} / \mathrm{L}, \mathrm{AST}-21.2 \pm 2.7 \mathrm{IU} / \mathrm{L}$, platelets $-267.3 \pm 22.6 \times$ $10^{9} / \mathrm{L}$. The biomarkers of liver fibrosis were as follows: APRI (index) $-0.19 \pm 0.01$, and FIB-4 (index) - $0.022 \pm 0.001$.

\section{DISCUSSION}

The world literature is lock of any studies about morphometric parameters of the stromal-parenchymal components of healthy liver of healthy infants from mothers with physiological pregnancy. The resulting morphometric data stromal-parenchymatous component of a normal liver (morphometric parameters of the number of hepatocytes, portal tracts and central veins, sinusoids, bile ducts, fibronectin, collagen I, III and IV types) can be used as control group in the study of variour pathological conditions of the liver of newborn children.

Liver biopsy is considered the «gold standard» for assessing the presence and severity of fatty liver and especially liver fibrosis. However, this procedure is invasive and is intended for children only in extreme cases [9]. Therefore, the scientific community has proposed two serum biomarkers APRI and FIB-4, which can reliably detect the presence and severity of liver fibrosis without the need for a traumatic liver biopsy procedure [10]. APRI and FIB-4 liver fibrosis biomarkers are simple and easy to use, and there are no complex components in formulas that requireds to calculate them. To calculate both biomarkers of fibrosis, it is necessary to register the ALT, AST, and platelet counts in the blood plasma (routine total blood count and hepatic metabolic panel), and for the FIB-4 biomarker, one must have the information about patient's age.

Biomarkers of liver fibrosis have been well studied in adults, however, there are lock of any studies about biomarkers of liver fibrosis in healthy newborns from healthy mothers with physiological pregnancy, so this study presents the data about APRI and FIB-4 for the first time.

\section{CONCLUSIONS}

The presented morphometric data of stromal-parenchymal component of the normal liver of healthy infants from healthy mothers with physiological pregnancy (morphometric parameters of number of hepatocytes, portal tracts and central veins, sinusoids, bile ducts, fibronectin, collagen I, III and IV types), and biomarkers of liver fibrosis APRI and FIB-4 can be used as a control group in the study of any pathological conditions in the liver of newborns.

\section{REFERENCES}

1. Finegold M.J. Pathology of the liver in children: Where would I (we) be without "Pepper"? Semin. Diagn. Pathol. 2016; 33(6): 367-376. DOI: 10.1053/j.semdp.2016.08.002.

2. Finegold M.J.Common diagnostic problems in pediatric liver pathology. Clin. Liver Dis. 2002; 6(2): 421-454.

3. Deirdre A. Kelly. Diseases of the liver and biliary system in children. Chichester, West Sussex; Hoboken, NJ: John Wiley \& Sons, Inc.; 2017; 636.

4. Avtandilov G.G. Osnovy kolichestvennoy patologicheskoy anatomii [The basics of quantitative pathological Anatomy]. Moscow: Medicine. 2002. (in Russian).

5. Pokorska-Śpiewak M., Stańska-Perka A., Popielska J. et al. Prevalence and predictors of liver disease in HIV-infected children and adolescents. Scientific Reports. Springer Nature. 2017; 7 (1): 12309. D01:10.1038/ s41598-017-11489-2.

6. Wai C. A simple noninvasive index can predict both significant fibrosis and cirrhosis in patients with chronic hepatitis C. Hepatology. 2003; 38(2): 518-526. D01:10.1053/jhep.2003.50346.

7. Sterling R. K., Lissen E., Clumeck N. et al. Development of a simple noninvasive index to predict significant fibrosis in patients with HIV/ HCV coinfection. Hepatology. 2006; 43(6): 1317-1325. D01:10.1002/ hep.21178.

8. Lin Z.-H., Xin Y.N., Dong Q.J. et al. Performance of the aspartate aminotransferase-to-platelet ratio index for the staging of hepatitis C-related fibrosis: An updated meta-analysis. Hepatology. 2011;53(3): 726-736. D0l:10.1002/hep.24105.

9. Dezsőfi A., Baumann U., Dhawan A. et al. Liver Biopsy in Children. Journal of Pediatric Gastroenterology and Nutrition. Ovid Technologies (Wolters Kluwer Health). 2015; 60(3): 408-420. D0I:10.1097/ MPG.0000000000000632.

10. Li Q., Ren, X., Lu, C. et al. Evaluation of APRI and FIB-4 for noninvasive assessment of significant fibrosis and cirrhosis in HBeAg-negative CHB patients with ALT $\leq 2$ ULN. Medicine. 2017; 96(12): e6336. D0I:10.1097/ MD. 0000000000006336 . 
This work is a fragment of a comprehensive research program of the Human Anatomy Department of the V.N. Karazin Kharkiv National University: "The impact of maternal pathology on the fetal and newborn organism development". State Registration Number: 0117U004838. This work was funded according to the plan of that program. The authors did not receive any financial support from the manufacturers of medical instruments and drugs.

\section{ORCID and contributionship.}

Sergey O. Sherstiuk: 0000-0001-6062-7962 A,E,F

Stanislav I. Panov: 0000-0001-9264-0088 ${ }^{B, C, D}$

Tetiana I. Liadova: 0000-0002-5892-2599 B,C,E

Oleksij I. Tsivenko: 0000-0003-0839-9768 ${ }^{B, C, E}$

Liudmila L. Sherstiuk: 0000-0002-2993-2843 ${ }^{\text {B,C,E }}$

\section{Conflict of interest:}

The Authors declare no conflict of interest.

\section{CORRESPONDING AUTHOR}

Stanislav I. Panov

V. N. Karazin Kharkiv National University

6 Svobody Sq., 61022 Kharkiv, Ukraine

tel:+38 099 460-39-94,

e-mail:StanislavPanov@karazin.ua

Received: 22.09.2019

Accepted: 17.07 .2020

A - Work concept and design, B - Data collection and analysis, C - Responsibility for statistical analysis,

D-Writing the article, $\mathbf{E}$-Critical review, $\mathbf{F}$ - Final approval of the article 